\title{
L'alliance pédagogique en supervision clinique : une étude qualitative en sciences de la santé
}

\section{Educational alliance during clinical supervision: a qualitative study in health sciences}

\author{
Luc CÔTÉ ${ }^{1, *}$, Erik BRETON²${ }^{2}$, Dominique BOUCHER ${ }^{3}$, Élisabeth DÉRY ${ }^{4}$, et Jean-François ROUX ${ }^{5}$ \\ ${ }^{1}$ Département de médecine familiale et de médecine d'urgence, Faculté de médecine, Université Laval, Québec, Canada \\ 2 Centre de recherche en éducation des sciences de la santé, Faculté de médecine, Université Laval, Québec, Canada \\ 3 Département de réadaptation, Programme d'ergothérapie, Faculté de médecine, Université Laval, Québec, Canada \\ ${ }^{4}$ Département de réadaptation, Programme d'orthophonie, Faculté de médecine, Université Laval, Québec, Canada \\ ${ }^{5}$ Département de réadaptation, Programme de physiothérapie, Faculté de médecine, Université Laval, Québec, Canada
}

Manuscrit reçu le 11 mai 2018 ; commentaires éditoriaux formulés aux auteurs le 8 novembre 2018 ; accepté pour publication le 11 novembre 2018

\begin{abstract}
Résumé - Contexte: La notion d'«alliance pédagogique»a été proposée comme cadre conceptuel de la rétroaction axée sur le dialogue en supervision. Or, nous ne savons pas comment les superviseurs et les stagiaires en sciences de la santé se la représentent et en font l'expérience en supervision clinique. Objectifs : L'étude qualitative visait à décrire la signification de l' «alliance pédagogique » pour les superviseurs et les stagiaires en sciences de la santé, les facteurs contribuant ou non à son développement, ainsi que les stratégies de gestion des alliances pédagogiques plus difficiles qui furent vécues. Méthodes : Des entrevues téléphoniques semi-dirigées ont été réalisées auprès d'un échantillonnage intentionnel de 45 superviseurs et de 29 stagiaires en fin de programme provenant de médecine familiale, ergothérapie, orthophonie et physiothérapie de l'Université Laval (Québec, Canada). Les données transcrites sous forme de verbatim ont été codifiées et ont fait l'objet d'une analyse thématique de contenu inductive. Résultats : La notion d' «alliance pédagogique » était nouvelle pour la majorité des participants. Ses composantes sont la collaboration, la confiance, le respect et la communication. Lorsque celle-ci a été vécue plus difficilement, les superviseurs et les stagiaires ont joué un rôle actif dans leur décision de discuter ouvertement ou non de la problématique en supervision, mais pour des raisons parfois différentes. Conclusion : L'alliance pédagogique est un processus interactionnel complexe qui est influencé par plusieurs facteurs. Les résultats de cette étude serviront à mieux saisir sa signification et ses implications, ainsi qu'à élaborer des activités de formation, autant pour les superviseurs que pour les stagiaires.
\end{abstract}

Mots clés : alliance pédagogique, supervision clinique, rétroaction, sciences de la santé, recherche qualitative

\begin{abstract}
Background: A few years ago, the "educational alliance" framework was proposed to highlight the role of context and relationship in the feedback process. However, we don't know how supervisors and trainees in different health sciences programs perceive and experience this educational alliance. Objectives: This qualitative study aimed to describe how supervisors and trainees in different health sciences programs perceive the educational alliance, the factors contributing or not to a quality educational alliance and how the strategies to restore it in difficult supervisions they have experienced. Methods: We conducted semi-structured, one-onone phone interviews with a purposeful sample of 45 supervisors and 29 senior trainees from family medicine, occupational therapy, physiotherapy, and speech therapy programs at Université Laval, Quebec, Canada. Transcribed data were coded and analyzed using an inductive qualitative content analysis. Results: The concept of "educational alliance" was new for most participants. The notions of collaboration, mutual trust, respect, and honest communication were identified as the pillars of this alliance. When supervisors and trainees
\end{abstract}

\footnotetext{
*Correspondance et offprints: Luc CÔTÉ, Département de médecine familiale et de médecine d'urgence, Faculté de médecine, pavillon Ferdinand-Vandry, bureau 2476, 1050 avenue de la Médecine, Université Laval, G1V 0A6 Québec, Canada.

Mailto: luc.cote@fmed.ulaval.ca.
} 
seek to overcome difficulties and restore an unsatisfactory educational alliance, they played an active role in this decision-making process, but have different reasons for deciding whether or not to raise the issue during supervision. Conclusions: The educational alliance is a complex interactional process influenced by different factors. Our findings will be helpful in a better understanding of its meaning and impact and in planning faculty development activities for supervisors and clinical students.

Keywords: educational alliance, clinical supervision, feed-back, health sciences, qualitative research

\section{Introduction}

La supervision clinique occupe une place importante dans les programmes de formation des professionnels de la santé. En effet, elle contribue au développement des compétences et de l'identité professionnelle du stagiaire, en plus d'assurer la qualité des soins et des services [1-2]. La supervision «désigne l'intervention pédagogique d'un enseignant clinicien lorsque celui-ci fournit une rétroaction à un étudiant après l'avoir directement observé dans une tâche professionnelle, par exemple auprès d'un patient, d'une famille ou d'une équipe de soins (supervision «directe»), ou à partir d'un résumé de la situation qu'en fait l'étudiant (supervision «indirecte») »[3]. Dans ce texte, nous emploierons le terme générique «stagiaire» pour faire référence à l'étudiant en cours de formation professionnelle en santé, quels que soient la discipline, la profession ou le programme concernés. De la même manière, nous emploierons le terme de «superviseur » pour faire référence à l'enseignant clinicien qui supervise le stagiaire dans un milieu clinique.

\section{Contexte et problématique}

La rétroaction est au cour de la supervision clinique. Outre qu'elle aide le stagiaire à gérer les situations cliniques, elle le renseigne sur son efficience, en précisant ses forces et ses limites, le soutient dans l'identification de ses besoins d'apprentissage pour progresser, le guide vers les stratégies et ressources adéquates pour répondre à ses besoins et finalement, le motive à s'engager dans des activités d'apprentissage pertinentes [4].

Or, depuis une dizaine d'années, plusieurs études en éducation médicale [5-14] ont démontré que la rétroaction est souvent problématique en raison des divergences, voire des tensions, entre les superviseurs et les stagiaires quant à la fréquence de la rétroaction, sa qualité, sa pertinence et son efficacité. Par exemple, alors que les superviseurs rapportent échanger des rétroactions constructives selon une fréquence régulière, les stagiaires ont l'impression que la rétroaction reçue est globale, succincte et peu fréquente. De plus, l'ouverture et la réceptivité des stagiaires aux commentaires des superviseurs seraient souvent mitigées selon les superviseurs. Ces études ont confirmé que la rétroaction est un processus interactionnel complexe, qui ne peut se réduire à une conception purement comportementaliste - ou béhavioriste- de la rétroaction dans laquelle le superviseur «émetteur » transmet ses commentaires au stagiaire « récepteur».

\section{Cadre conceptuel}

C'est dans ce contexte que des auteurs ont proposé de faire une relecture de la rétroaction à partir de la perspective socioculturelle de la rétroaction. Celle-ci stipule que la rétroaction est un processus hautement collaboratif qui est influencé par divers facteurs : ceux se rapportant au superviseur, par exemple, sa crédibilité ; les facteurs qu'on associe au stagiaire, par exemple, son ouverture aux commentaires; ceux s'appliquant à la relation superviseur-stagiaire, par exemple la qualité de la communication; ainsi que les facteurs en lien avec la culture du milieu dans lequel les rétroactions ont lieu, par exemple un milieu qui valorise ou non l'importance de la rétroaction constructive. Ainsi, selon cette perspective, on définit la rétroaction comme un dialogue entre le superviseur et le stagiaire, dont le but est non seulement de commenter l'efficience du stagiaire quant à ses points forts et aux aspects à améliorer, mais de le soutenir dans le développement de ses compétences en l'aidant à identifier des objectifs réalistes pour progresser [15-17].

Récemment, Telio et al. [18] ont proposé la notion d' «alliance pédagogique » comme cadre conceptuel pour mieux comprendre la rétroaction axée sur le dialogue lors de la supervision. À partir des écrits de Bordin sur «l'alliance thérapeutique » ou «alliance de travail » [19] et sur «l'alliance en supervision» [20], ces auteurs considèrent que pour qu'il y ait un réel dialogue lors de la rétroaction, l'alliance pédagogique doit être de qualité, ce qui signifie : 1) que le superviseur et le stagiaire aient une compréhension commune des objectifs de la rétroaction; 2) qu'ils soient d'accord sur les moyens de les atteindre et ; 3) qu'ils développent un lien de confiance pendant la rétroaction. Le point de vue du stagiaire serait particulièrement important lors de l'évaluation de l'alliance pédagogique, car celui-ci est l'objet de la rétroaction. L'alliance pédagogique n'est pas une situation stable. À l'instar de l'alliance thérapeutique, dans les faits, elle comporte inévitablement des fluctuations plus ou moins importantes dites normales, même si les trois conditions proposées par Telio et al. sont satisfaites. De fait, la présence de problèmes relationnels jugés significatifs par l'un ou l'autre des partenaires peut fragiliser cette alliance et même mener à sa rupture, en raison de divers facteurs personnels, interactionnels et contextuels [21].

Une alliance pédagogique de qualité a des impacts pédagogiques majeurs en supervision. Ainsi, Hauer et al. $[22,23]$ ont identifié que la qualité de la relation entre le 
superviseur et le stagiaire figure parmi les principaux facteurs contribuant à la confiance qu'accorde le superviseur au stagiaire. De même, Telio et al. [12] ont constaté que la crédibilité des superviseurs, telle qu'elle est perçue par des stagiaires en psychiatrie, repose non seulement sur l'expertise clinique de leur superviseur, mais aussi sur la perception de leur contribution au développement d'une alliance pédagogique positive lors de la rétroaction. Enfin, Bowen et al. [13] ont démontré que la qualité de l'alliance pédagogique influence positivement la perception que les stagiaires en médecine ont à l'égard de la rétroaction de qualité.

À notre connaissance, aucune étude n'a porté spécifiquement sur l'alliance pédagogique lors de la rétroaction en supervision clinique. Les études mentionnées ci-dessus, réalisées en médecine, ont associé cette notion à d'autres dimensions, par exemple à la crédibilité du superviseur mais non spécifiquement à l'alliance pédagogique pendant la rétroaction. Nous ne savons donc pas comment les superviseurs et les stagiaires, que ce soit en médecine ou dans d'autres programmes en sciences de la santé, perçoivent et font l'expérience de cette alliance.

\section{Objectifs}

Dans ce contexte, le présent travail a cherché à explorer, dans le cadre d'une étude qualitative, la signification et les expériences liées à l'alliance pédagogique lors de la rétroaction en stage. Nous nous sommes intéressé plus spécifiquement aux facteurs contribuant au développement d'alliances pédagogiques jugées satisfaisantes et insatisfaisantes, ainsi qu'aux stratégies pour gérer les alliances pédagogiques insatisfaisantes. Nos questions de recherche étaient les suivantes:

- Que signifie «l'alliance pédagogique» pendant la rétroaction en supervision clinique pour les superviseurs et les stagiaires en sciences de la santé?

- Quels facteurs contribuent, selon les expériences vécues, au développement d'alliances pédagogiques satisfaisantes?

- Est-ce que les superviseurs et les stagiaires ont vécu à une ou plusieurs occasions des alliances pédagogiques insatisfaisantes et si oui, comment ont-ils géré la problématique?

\section{Méthodes \\ Devis}

Nous avons réalisé une étude qualitative selon les principes de l'approche socioconstructiviste de la théorisation ancrée $[24,25]$. En résumé, cette approche méthodologique vise une meilleure compréhension d'un phénomène complexe à partir d'une analyse inductive et systématique des données issues du terrain de recherche au fur et à mesure du déroulement de l'étude.

\section{Recrutement des participants}

L'étude a été réalisée auprès de superviseurs et de stagiaires en fin de formation provenant des programmes de médecine familiale, d'ergothérapie, d'orthophonie et de physiothérapie de l'Université Laval (Québec, Canada) entre avril 2016 et septembre 2017. Ces programmes ont été sélectionnés en raison de leur intérêt pour la supervision clinique, intérêt qui se traduit par l'offre pour les superviseurs d'activités de formation se rapportant à la supervision clinique.

Le recrutement s'est fait en deux étapes. Pour la première étape, nous avons constitué une liste d'environ 50 superviseurs et de 35 stagiaires en fin de formation provenant des programmes ciblés. Nous avons dressé une liste de participants potentiels en nous appuyant sur les critères suivants: (1) la diversité des superviseurs (type de formation, genre, années d'expérience en supervision, milieux de stage variés); (2) la diversité des stagiaires (programme de formation, genre, expériences de stage variées avec différents superviseurs, d'où l'importance de sélectionner des stagiaires en fin de formation). La diversité des participants est reconnue comme étant un des critères de scientificité en recherche qualitative, car elle contribue à bien saisir la problématique à l'étude à partir de plusieurs expériences, points de vue et perspectives [26]. De plus, elle constitue une forme de triangulation des données [27]: les informations proviennent d'au moins deux sources complémentaires différentes. Celle-ci aide à atteindre la saturation des données [28], ce qui signifie que l'ajout de nouvelles informations n'ajouterait rien de neuf à celles déjà recueillies.

$\mathrm{Au}$ cours de la deuxième étape, une lettre de sollicitation personnalisée, accompagnée d'un formulaire d'information et de consentement qui précisait notamment les mesures prises pour assurer la confidentialité des informations, fut envoyée par courriel aux participants potentiels. Pour être sélectionnés, ceux-ci devaient nous retourner le formulaire de consentement de participation dûment signé. Toutes les personnes ayant signé ce formulaire ont été acceptées dans l'étude.

\section{Recueil des données}

Le recueil des données s'est fait lors d'une entrevue individuelle téléphonique semi-dirigée (voir le guide d'entrevue à l'Annexe A). Le chercheur principal (LC) a mené toutes les entrevues avec les superviseurs, alors que celles avec les stagiaires l'ont été, soit par le chercheur principal ( $50 \%$ des entrevues), soit par le professionnel de recherche (EB), ces deux personnes détenant une longue expérience dans la conduite d'entrevues dans le cadre d'études qualitatives. Les entrevues ont été enregistrées en audio en vue de la codification et de l'analyse.

\section{Codification et analyse des données}

Les entrevues ont été transcrites intégralement, puis anonymisées afin que les chercheurs ne puissent pas reconnaître l'identité des participants. La codification inductive s'est faite en suivant la démarche habituelle d'une analyse thématique de contenu [29] et en respectant le principe de la triangulation des chercheurs, selon lequel au moins deux personnes codifient les données de manière 
Tableau I. Données sociodémographiques et profil des participants.

\begin{tabular}{|c|c|c|c|c|}
\hline \multirow[t]{2}{*}{ Participants $(n=74)$} & \multicolumn{4}{|c|}{ Programmes } \\
\hline & Ergothérapie $(n=20)$ & Médecine familiale $(n=18)$ & Orthophonie $(n=17)$ & Physiothérapie $(n=19)$ \\
\hline Superviseurs $(n=45)$ & $n=14$ & $n=11$ & $n=10$ & $n=10$ \\
\hline $37 \mathrm{~F}(82 \%) / 8 \mathrm{H}$ & $13 \mathrm{~F}(93 \%) / 1 \mathrm{H}$ & $8 \mathrm{~F}(73 \%) / 3 \mathrm{H}$ & $10 \mathrm{~F}(100 \%)$ & $6 \mathrm{~F}(60 \%) / 4 \mathrm{H}$ \\
\hline $\begin{array}{l}\text { Expérience en supervision } \\
\text { Moyenne : } 13 \text { ans } \\
\text { (Min. :1-Max. :38) } \\
\text { Durée des entrevues } \\
\text { Moyenne : } 37 \text { min. } \\
\text { (Min. :14-Max. :49) }\end{array}$ & 16 ans $(4-34)$ & 12 ans $(2-32)$ & 6 ans $(1-15)$ & 13 ans $(2-38)$ \\
\hline Stagiaires $(n=29)$ & $n=6$ & $n=7$ & $n=7$ & $n=9$ \\
\hline $\begin{array}{l}23 \mathrm{~F}(79 \%) / 6 \mathrm{H} \\
\text { Durée des entrevues } \\
\text { Moyenne : } 32 \text { min. } \\
\text { (Min. : } 12-\text { Max. :47) }\end{array}$ & $5 \mathrm{~F}(83 \%) / 1 \mathrm{H}$ & $5 \mathrm{~F}(71 \%) / 2 \mathrm{H}$ & $6 \mathrm{~F}(86 \%) / 1 \mathrm{H}$ & $7 \mathrm{~F}(78 \%) / 2 \mathrm{H}$ \\
\hline
\end{tabular}

$\mathrm{H}$ : hommes; $\mathrm{F}$ : femmes

indépendante, puis partagent leurs résultats. Concrètement, toutes les entrevues ont été codifiées par LC et EB, alors que pour $40 \%$ des entrevues, un troisième membre de l'équipe de recherche a aussi été impliqué dans la codification (DB, ED ou JFR). Tous les membres de l'équipe de recherche ont participé aux étapes itératives subséquentes de l'analyse des données. Le logiciel NVIVO 11 a servi à la gestion des données.

\section{Résultats}

\section{Données sociodémographiques et profil des participants}

Au total, 74 personnes ont participé, soit 45 superviseurs et 29 stagiaires, dont une majorité de femmes, autant chez les superviseurs que chez les stagiaires, ce qui est représentatif de la population de ces programmes (Tableau I).

Les résultats seront présentés en trois sections : (1) la signification et les indicateurs de l'alliance pédagogique; (2) les facilitateurs et les obstacles au développement d'alliances pédagogiques satisfaisantes et; (3) la gestion des alliances pédagogiques insatisfaisantes.

\section{Signification et indicateurs de l'alliance pédagogique}

Cette notion était nouvelle pour la très grande majorité des participants, autant chez les superviseurs que chez les stagiaires. Les participants l'ont spontanément associée à la «bonne relation de supervision » en général, au «bon climat de supervision et d'apprentissage en stage», sans l'appliquer systématiquement à la rétroaction :

«...l'alliance pédagogique...hum, j'imagine que c'est la bonne entente entre le superviseur et le stagiaire pendant le stage... c'est difficile de parler de ça sans parler d'autres aspects de la supervision parce que tout est lié non?» (superviseur 4).

Pour la majorité des participants, l'alliance pédagogique est une relation comportant quatre caractéristiques indissociables: la collaboration, la confiance mutuelle, le respect mutuel et la communication (voir Tableau II).

Les principales métaphores dont les participants ont fait usage pour en parler sont: «un lien qu'on tisse; une construction à deux; un cheminement pédagogique; une entente contractuelle; une discussion où on est sur la même longueur d'ondes, où les astres s'alignent; une communication fluide comme une rivière». Quelques superviseurs ont spontanément associé l'alliance pédagogique à l'alliance thérapeutique, en insistant sur l'importance du lien de confiance lors des échanges pendant la rétroaction :

«...Je trouve qu'on peut faire le parallèle avec l'alliance thérapeutique en clinique. Si la confiance n'est pas là, y aura pas d'alliance possible. C'est la même chose en supervision, surtout pendant les périodes de rétroaction : il faut qu'on sente que la confiance est là, qu'on peut se fier à ce que l'autre nous dit. Je ne vois pas comment un stagiaire serait ouvert aux commentaires du superviseur s'il ne lui fait pas confiance. C'est la même chose pour le superviseur. » (superviseur 3).

L'alliance pédagogique jugée satisfaisante se manifeste, autant pour les superviseurs que pour les stagiaires, par le goût et le plaisir de travailler ensemble en supervision, plus spécifiquement par la facilité des échanges lors des périodes de rétroaction. Le sentiment d'être utile lors des rétroactions a aussi été rapporté par les superviseurs, alors que pour les stagiaires, la perception du sentiment de soutien et d'encouragement de la part du superviseur, ainsi que le sentiment d'être perçus par ce-dernier comme des collègues et non des étudiants juniors sont particulièrement importants pour que l'alliance soit satisfaisante.

Voici deux exemples provenant de stagiaires :

«Quand tu sens que tu as du plaisir et de la complicité à travailler ensemble, surtout quand tu discutes les cas, je pense que ça veut dire qu'il y a une bonne alliance entre le superviseur et toi. » (stagiaire 18). 
Tableau II. Récapitulatif des thèmes associés à «l'alliance pédagogique » rapportés par les superviseurs et les stagiaires participant à l'étude.

\begin{tabular}{lll}
\hline L'alliance pédagogique est une relation de & Superviseurs (\% de 45) & Stagiaires (\% de 29) \\
\hline $\begin{array}{l}\text { Collaboration/partenariat/équipe } \\
\text { (Le superviseur et le stagiaire travaillent activement et en complémentarité } \\
\text { à l'atteinte d'objectifs cliniques et pédagogiques partagés) }\end{array}$ & $40(89 \%)$ & $25(86 \%)$ \\
$\begin{array}{l}\text { Confiance mutuelle } \\
\text { (Le stagiaire reconnaît l'expertise du superviseur et son désir de l'aider, }\end{array}$ & $38(84 \%)$ & $24(83 \%)$ \\
$\begin{array}{l}\text { alors que le superviseur reconnaît la motivation du stagiaire à progresser } \\
\text { et à demander de l'aide lorsque c'est plus difficile) }\end{array}$ & $36(80 \%)$ & $24(83 \%)$ \\
$\begin{array}{l}\text { Respect mutuel } \\
\text { (Même si la relation est hiérarchique sur le plan pédagogique, } \\
\text { elle est égalitaire sur le plan humain. L'écoute, l'ouverture au } \\
\text { point de vue de l'autre et la politesse sont jugés essentiels). }\end{array}$ & \\
$\begin{array}{l}\text { Communication } \\
\text { (Les messages sont clairs, autant les points positifs sur la } \\
\text { performance que les aspects à améliorer. Le superviseur et le } \\
\text { stagiaire sont honnêtes dans leurs échanges) }\end{array}$ & $35(78 \%)$ & $21(76 \%)$ \\
\hline
\end{tabular}

a Les nombres et les pourcentages sont présentés uniquement à titre indicatif.

«Je sais que le superviseur et moi on a une bonne alliance quand on se fait confiance: il montre qu'il a confiance en moi en me laissant de l'autonomie et moi je lui montre que j'ai confiance en lui en lui parlant des choses qui sont plus difficiles pour moi dans mon apprentissage. » (stagiaire 7).

\section{Facilitateurs et obstacles au développement d'alliances pédagogiques satisfaisantes \\ Facilitateurs}

Les superviseurs et les stagiaires ont été en mesure d'évoquer une ou plusieurs expériences d'alliances pédagogiques satisfaisantes en supervision en général et lors de la rétroaction en particulier. Ils ont aussi exprimé qu'en rétrospective, la majorité des expériences qu'ils avaient vécues en tant que superviseur ou stagiaire avaient été globalement positives. Les facteurs ayant facilité le développement d'une alliance satisfaisante correspondaient fidèlement aux quatre caractéristiques de l'alliance pédagogique présentées au tableau II, soit : la motivation et l'habileté du superviseur et du stagiaire à établir un climat de collaboration, à se faire confiance, à se respecter et à bien communiquer. Les participants ont jugé que tous ces facteurs relationnels étaient essentiels pour tirer le plein potentiel de la supervision, surtout lors des périodes de rétroaction et qu'ils étaient tous importants, quels que soient les contenus discutés.

À ces facteurs s'ajoute l'habileté du superviseur à échanger de la rétroaction constructive, c'est-à-dire qui est utile à l'apprentissage. En effet, pour la majorité des participants, davantage chez les stagiaires, le superviseur joue un rôle primordial à ce niveau. Le verbatim suivant résume bien plusieurs propos entendus pendant les entrevues sur le rôle du superviseur lors de la rétroaction :
«...Quand je parle de ça (rétroaction constructive), ce que je veux dire c'est que le superviseur prend le temps d'expliquer sa pensée au stagiaire, pourquoi il dit telle ou telle chose et il le fait toujours d'une manière respectueuse en s'assurant que le stagiaire comprend. Par son attitude, il donne le message au stagiaire qu'il est important qu'il participe, qu'il donne son point de vue même s'il n'est pas d'accord avec le superviseur. Ça ne se fait pas à sens unique une bonne rétroaction, mais c'est le superviseur qui doit avoir les bonnes antennes et les bonnes façons de faire pour que le stagiaire et lui forment une équipe. » (superviseur 11).

Finalement, l'ouverture du stagiaire aux commentaires du superviseur semble aussi un facteur incontournable au développement d'une alliance pédagogique satisfaisante lors de la rétroaction, car ce thème a été rapporté par plusieurs participants, davantage par les superviseurs.

\section{Obstacles}

Au total, neuf superviseurs $(20 \%)$ ont rapporté avoir déjà vécu, depuis qu'ils supervisent, au moins une alliance pédagogique moins satisfaisante ou plus difficile avec leur stagiaire, alors que 14 superviseurs (31\%) se souvenaient en avoir vécu une ou plusieurs lorsqu'ils étaient euxmêmes stagiaires. Dans le groupe des stagiaires, sept d'entre eux $(24 \%)$ en auraient vécu. Dans les deux groupes, les alliances jugées moins satisfaisantes ont toujours eu un impact émotionnel significatif, impact encore plus important selon l'ampleur des difficultés relationnelles vécues entre le superviseur et le stagiaire:

«...C'est arrivé seulement une fois dans un stage de... mais je m'en souviens comme si c'était hier tellement ça a été difficile... Il m'arrivait souvent de pleurer en 
rentrant chez-moi le soir tellement c'était pénible avec ce superviseur-là... Mon conjoint se souvient certainement lui aussi de ce stage-là.» (stagiaire 3).

«...Je me souviens en particulier d'un stagiaire avec qui ça ne fonctionnait pas, on n'était pas sur la même longueur d'ondes, on était souvent en désaccord... je ne me sentais pas bien avec lui parce que j'avais l'impression qu'il ne me faisait pas confiance. J'étais en début de pratique et ça m'a brûlée, brûlée au point que je me suis sérieusement demandé si je continuerais à superviser des stagiaires. » (superviseur 31).

Les principaux indicateurs associés à une alliance pédagogique moins satisfaisante ou plus difficile rapportés par les participants en ayant vécu sont le sentiment d'inconfort à discuter avec l'autre, la communication difficile, la perception de ne pas être compris et un sentiment général de frustration et de fatigue. Le sentiment d'atteinte à l'estime de soi a aussi été mentionné par quelques stagiaires. Les métaphores utilisées par les superviseurs pour décrire ce type d'alliances furent: «quand le courant ne passe pas avec le stagiaire», quand le superviseur a l'impression de «pédaler dans le vide » ou de «marcher sur des œufs lors de la rétroaction». Celles des stagiaires étaient de «marcher sur une corde raide pendant la rétroaction », de «mettre les freins avant de parler au superviseur » et de «se sentir comme une conne ou une nouille pendant la rétroaction ».

Les obstacles à une alliance pédagogique moins satisfaisante lors de la rétroaction correspondent à l'absence d'un ou de plusieurs facteurs favorables mentionnés au tableau II, la communication difficile et le sentiment d'incompréhension contribuant de manière plus importante à la fragilité, voire à la rupture du lien de confiance et donc de l'alliance pédagogique.

\section{La gestion des alliances pédagogiques insatisfaisantes}

Les participants ayant vécu des alliances insatisfaisantes, soit comme superviseur $(20 \%)$, soit comme stagiaire $(24 \%)$, rapportent les avoir gérées selon la démarche présentée sur la figure 1.

Les participants analysent d'abord individuellement la situation problématique; cette analyse est parfois associée à la consultation de pairs ou d'autres personnes selon les besoins. S'ensuit une prise de décision: soit discuter ouvertement de la problématique d'alliance pédagogique avec le superviseur ou le stagiaire, soit, au contraire, ne pas en parler. Puis les moyens discutés pour améliorer l'alliance sont appliqués et ajustés si les moyens mis en place ne produisent pas les effets escomptés.

Plus spécifiquement, dans tous les cas, autant les superviseurs que les stagiaires ont rapporté avoir analysé individuellement la situation problématique en tentant d'abord de comprendre la ou les raisons explicatives des difficultés relationnelles ressenties pendant la rétroaction. Si certains ont analysé la situation d'une manière globale en évoquant par exemple un sentiment d'inconfort lors des discussions pendant la rétroaction, d'autres ont exploré une ou des hypothèses plus précises en vue de poser une sorte de «diagnostic pédagogique relationnel » à partir de facteurs potentiellement contributoires de la part du superviseur ou du stagiaire. Voici un exemple:

«...je me suis tellement posé de questions pour tenter de comprendre pourquoi c'était si difficile de discuter avec ce superviseur-là. Je me suis demandé si c'était causé par mon comportement, par nos personnalités différentes, par la fatigue, par la complexité des cas cliniques, etc. Finalement, je pense que le problème principal, c'est que mon superviseur ne savait pas comment être constructif dans ses rétroactions et que c'est pour cette raison que nos rencontres étaient si difficiles. » (stagiaire 27).

Lors de l'étape de l'analyse de la situation difficile avec le stagiaire, plusieurs superviseurs ont mentionné aussi avoir consulté un collègue et parfois le responsable facultaire des stages afin de discuter de leur représentation et d'explorer les interventions possibles. Quant aux stagiaires, ils en discutaient habituellement avec d'autres stagiaires, leur conjoint ou un ami et exceptionnellement avec le responsable facultaire des stages, s'ils jugeaient que la situation était suffisamment importante pour lui en parler. Il apparaît que plus la problématique relationnelle était vécue difficilement au plan affectif, plus la démarche de consultation se faisait de manière systématique.

À l'issue de l'analyse de la situation, les superviseurs décidaient ensuite s'ils discutaient ou non explicitement avec le stagiaire de ce qui semblait problématique en lien avec l'alliance pédagogique, notamment lors des échanges pendant la rétroaction. Trois critères principaux guidaient leur décision :

- l'importance perçue des difficultés relationnelles et de leur impact en supervision :

«...Ça dépend toujours de la situation. Si je trouve que c'est suffisamment important, par exemple si le climat de travail est difficile, si le stagiaire n'est pas ouvert à mes commentaires lors de la rétroaction, alors j'en parle, car cela a un impact sur la supervision. Sinon, je laisse faire. » (superviseur 37).

- le rapport coût-bénéfice d'en discuter ouvertement ou non :

«Dans ce temps-là, je me demande si ça vaut la peine que j'aborde avec le stagiaire le climat difficile qu'il y a dans nos échanges en supervision, comme l'inconfort pendant les rétroactions, si les chances qu'on arrive à un terrain d'entente et que ça s'améliore sont bonnes ou pas. J'analyse les avantages et les inconvénients d'en parler ou non.» (superviseur 15).

- la durée du stage et la fréquence des rétroactions. Par exemple : 
Analyse individuelle de la situation problématique

Consultation auprès de collègues et autres, au besoin

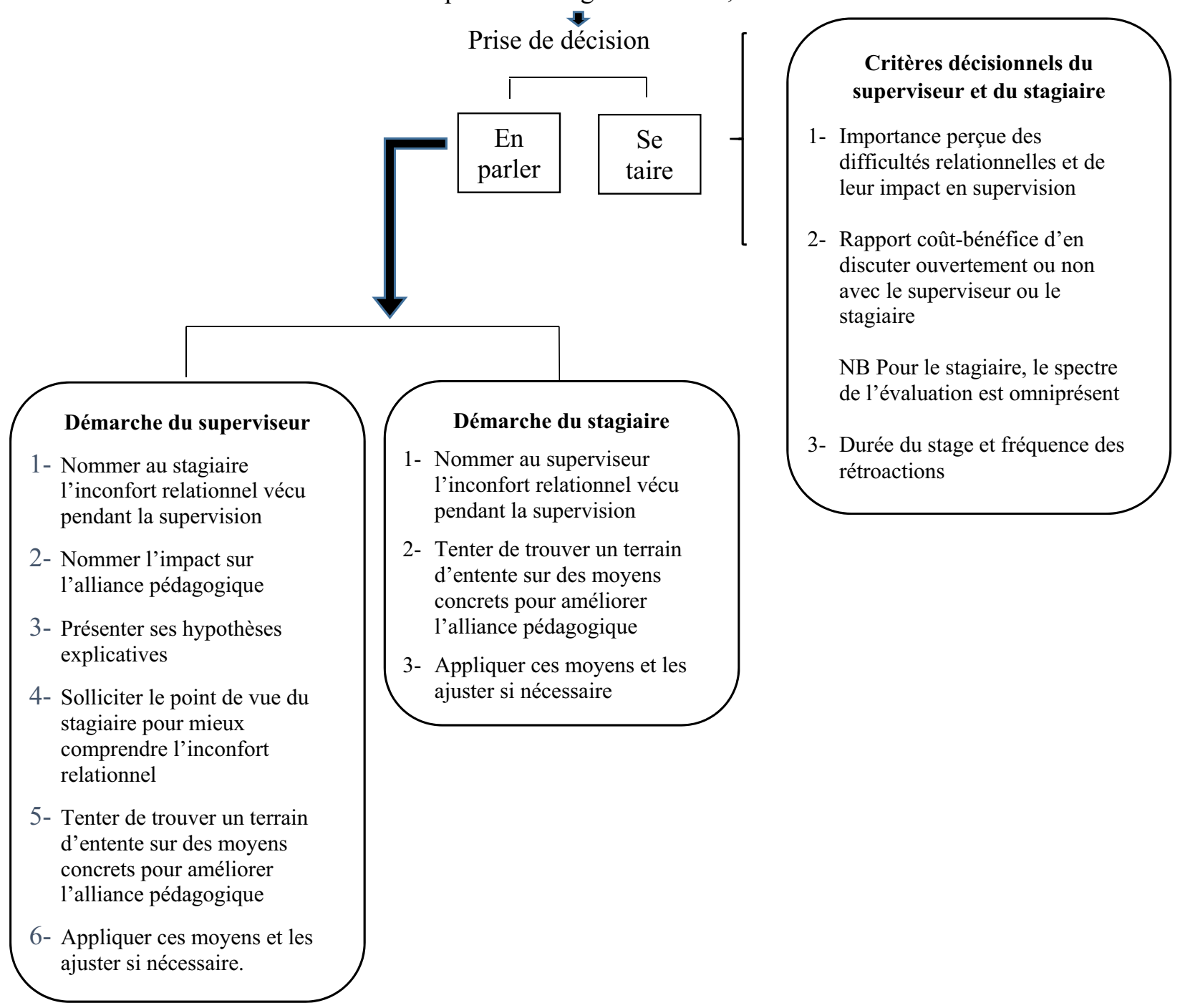

Figure 1. Processus de gestion des alliances pédagogiques insatisfaisantes, selon les superviseurs et les stagiaires, Université Laval.

«Si nous sommes plusieurs à superviser le même stagiaire et que je le supervise souvent, je vais lui en parler, surtout si le stagiaire fait un stage d'une durée de plusieurs semaines avec nous. Mais si le stagiaire n'est là que pour quelques jours ou que je ne le supervise que de temps en temps, je ne vais probablement pas lui en parler.» (superviseur 3).

Un superviseur nous a aussi dit que la présence d'une qualité relationnelle minimale avec le stagiaire représentait pour lui un facteur déterminant dans sa décision de discuter ou non des difficultés d'alliance avec lui :

«Je vais parler des problèmes d'alliance avec le stagiaire si je sens qu'il y a un minimum de lien positif ou d'alliance avec lui. Si je perçois que ce n'est pas présent, je ne vais pas en parler, car je risquerais d'envenimer la situation... Dans ce temps-là, même si ce n'est probablement pas la meilleure chose à faire, je vais mettre la priorité à bien gérer les cas cliniques, sans parler des difficultés qui sont vécues.» (superviseur 40).

Lorsque le superviseur décidait d'aborder explicitement les difficultés vécues avec le stagiaire, les principales stratégies d'intervention rapportées étaient les suivantes : (1) nommer l'inconfort ressenti (par exemple: fermeture du stagiaire aux commentaires du superviseur); (2) présenter au stagiaire une ou des hypothèses explicatives et leurs impacts en supervision (par exemple: impression que le stagiaire ne fait pas confiance au superviseur ce qui entraîne une méfiance du superviseur à l'égard du stagiaire); (3) solliciter le point de vue du stagiaire afin de développer la collaboration requise pour avoir un meilleur lien de confiance; (4) discuter de moyens concrets pour améliorer l'alliance, surtout le climat de confiance lors de la rétroaction; (5) 
les appliquer et les ajuster au besoin. Parmi les neuf superviseurs ayant rapporté des expériences insatisfaisantes se rapportant à l'alliance pédagogique, près de la moitié en aurait discuté avec le stagiaire, les autres ayant jugé préférable de ne pas le faire pour l'une ou l'autre des raisons mentionnées précédemment.

Quant aux stagiaires, le critère décisionnel principal les motivant à discuter ou non des difficultés vécues avec leur superviseur était la perception des conséquences positives et négatives que cela pourrait avoir sur l'évaluation de leur performance en stage et sur leur réputation comme stagiaire :

«...En parler? Mais jamais, jamais... J'aurais bien trop peur de passer pour une chialeuse et que ça nuise à mon évaluation. » (stagiaire 10).

«...Je vais en parler seulement si je pense que le superviseur va démontrer une certaine ouverture; c'est toujours un risque à prendre parce que certains superviseurs réagiraient très mal à aborder cette question et cela pourrait avoir des conséquences négatives pour moi dans les autres stages.» (stagiaire 26).

Parmi les stagiaires ayant vécu des difficultés d'alliance avec leur superviseur lors de la rétroaction, cinq sur sept $(71 \%)$ n'en auraient pas discuté ouvertement, par crainte des conséquences négatives sur leur évaluation. Toutefois, deux stagiaires ont mentionné avoir surmonté cette crainte et en avoir discuté avec leur superviseur parce qu'ils jugeaient que le mauvais climat relationnel nuirait trop à leurs apprentissages s'ils n'en parlaient pas :

«Une fois, j'ai dit à mon superviseur que je le trouvais blessant dans sa façon de me dire les choses. Il a eu l'air surpris et s'est excusé. Ça nous a permis de se parler de comment travailler ensemble pour que ce soit plus agréable et efficace. Je suis contente d'avoir fait ça parce que ça été beaucoup mieux par la suite et j'ai beaucoup appris à son contact. Cette fois-là, sans se le dire, on a parlé de notre alliance, du lien de confiance, je pense $\gg$ (stagiaire 6).

\section{Discussion}

La collaboration, la confiance et le respect mutuel, ainsi que la communication sont les fondements de l'alliance pédagogique, tant pour les superviseurs que pour les stagiaires. Bien que les pourcentages des résultats du tableau II aient été présentés à titre indicatif, on remarque que ces thèmes sont à $+\mathrm{ou}-3 \%$ convergents entre les superviseurs et les stagiaires. L'alliance a donc la même résonance de part et d'autre.

Ce résultat est cohérent avec les écrits en éducation, notamment en éducation médicale, et souligne l'importance de la qualité relationnelle en supervision clinique $[1,2,30,31]$, plus spécifiquement pour que la rétroaction soit un véritable dialogue entre le superviseur et le stagiaire [15-18]. Ils mettent aussi en évidence l'importance d'une forme de synchronisme entre le superviseur et le stagiaire pendant la rétroaction, ce qui correspond à la métaphore de la «danse du tango » qu'utilise Bing-You [32] en référence à la dimension interactionnelle inhérente à la rétroaction, selon la perspective socioculturelle de la supervision clinique.

Certains résultats ont attiré notre attention. D'abord, alors que Telio et al. [18] proposent la notion de l'alliance pédagogique comme cadre conceptuel pour comprendre la rétroaction comme étant un échange axée sur le dialogue, les participants en font une application plus globale. En effet, ils appliquent cette notion au confort relationnel entre le superviseur et le stagiaire pendant l'ensemble des activités de supervision en stage et plus particulièrement lors des périodes de rétroaction. Cette représentation est pertinente à la lumière du cadre conceptuel de Hauer et al. [22] qui stipule que la qualité relationnelle entre le superviseur et le stagiaire est un des facteurs de développement du lien de confiance en supervision, ce qui inclut évidemment les périodes de rétroaction, sans s'y limiter.

Par ailleurs, lorsque qu'ils sont questionnés sur les alliances pédagogiques insatisfaisantes, $20 \%$ des superviseurs et $24 \%$ des stagiaires ont exprimé en avoir vécu. On peut interpréter ce résultat comme une excellente nouvelle, au sens où l'alliance pédagogique semble de qualité dans la plupart des cas. Mais ce résultat est peutêtre influencé par un biais de désirabilité sociale qui pourrait engendrer une sous-estimation des insatisfactions rapportées. En effet, certains participants ont pu se sentir en situation d'inconfort pour relater des malaises, voire des difficultés, car en faire état pouvait laisser croire qu'ils n'avaient pas su développer une alliance de qualité, ce qui pouvait susciter un sentiment d'incompétence relationnelle en supervision.

L'autre résultat qui nous a interpellés a trait au fait que parmi les superviseurs ayant rapporté des alliances pédagogiques plus difficiles et insatisfaisantes, la moitié (4/8) n'en n'ont pas discuté avec leur stagiaire, alors que chez ces derniers, la majorité $(5 / 7)$ a décidé de taire au superviseur les difficultés relationnelles vécues avec lui. On constate que les superviseurs et les stagiaires jouent un rôle très actif dans ce processus décisionnel, mais que les raisons sous-jacentes à leur décision d'en parler ou non sont parfois différentes, par exemple la crainte des conséquences qu'en parler avec le superviseur pourrait avoir pour les stagiaires sur leur évaluation ou sur leur réputation. Cet élément pourrait expliquer en bonne partie la possible difficulté de la mise en place ou du maintien de l'alliance pédagogique. En effet, bien que la rétroaction soit perçue comme un dialogue visant la progression du stagiaire et que la collaboration, la confiance, le respect et la communication caractérisent l'alliance pédagogique, il n'en demeure pas moins que la composante évaluative, qu'elle soit formative ou sanctionnante, demeurera inévitablement en toile de fond dans la supervision clinique, davantage pour les stagiaires. Nous pensons d'ailleurs que la qualité de l'alliance pédagogique permet non pas d'évacuer cette composante évaluative pendant la supervision, mais qu'elle aide 
probablement à la gérer plus facilement en raison de la qualité du lien de confiance entre le superviseur et le stagiaire.

Cette étude comporte des limites. Même si elle a été réalisée auprès de plusieurs superviseurs et de stagiaires issus de programmes différents en sciences de la santé, ils provenaient d'une seule université francophone canadienne. Il faudrait voir si ces résultats s'appliquent à d'autres programmes et à d'autres universités où les cultures de la rétroaction et de la supervision clinique sont peut-être différentes. De plus, l'échantillonnage composé d'informateurs-clés a été constitué par les membres de l'équipe de recherche qui incluait des coordonnateurs universitaires de stage. Il est possible que les personnes ayant accepté de participer étaient déjà sensibilisées à l'importance de la qualité de l'alliance pédagogique en supervision même si ce terme était nouveau pour plusieurs et que d'autres superviseurs et stagiaires provenant des mêmes programmes auraient peut-être répondu autrement à nos questions.

Parmi les pistes de recherche futures, nous suggérons que des études du même type soient réalisées dans d'autres programmes et institutions. De plus, nous n'avons pas comparé les résultats selon les programmes. De même, l'utilisation d'une autre technique de recueil des données qualitatives permettrait peut-être d'enrichir les perspectives sur l'alliance pédagogique, par exemple, des entrevues de groupe conjointes entre superviseurs et stagiaires. Par ailleurs, nous ne savons pas si et comment le niveau d'expérience des superviseurs influence le développement d'alliances pédagogiques satisfaisantes, ni la gestion d'alliance plus difficiles. Finalement, tel que proposé par Wearne [33], il serait intéressant de savoir si et comment l'alliance pédagogique influence le contenu de la rétroaction.

\section{Conclusion}

Cette étude qualitative a permis de mieux comprendre comment les superviseurs et les stagiaires provenant de quatre programmes en sciences de la santé de l'Université Laval se représentent et ont vécu l'alliance pédagogique lors de la rétroaction en supervision clinique. Les deux partenaires de cette alliance ont été consultés permettant ainsi d'avoir une représentation riche sur le sujet.

\section{Contributions}

Luc Côté, chercheur principal, a élaboré la version initiale $\mathrm{du}$ protocole de recherche et a coordonné l'ensemble du projet. Il a été responsable du recrutement des superviseurs et des stagiaires en médecine familiale, a participé au recueil, à la codification et à l'analyse de l'ensemble des données. Il a rédigé la version initiale du manuscrit, puis, en collaboration, les versions successives menant à la production de la version finale du manuscrit. Erik Breton, professionnel de recherche au Centre de recherche en éducation des sciences de la santé à la Faculté de médecine de l'Université Laval au moment du déroulement projet, a participé à l'élaboration du protocole de recherche, au recueil, à la codification et à l'analyse de l'ensemble des données. Il a contribué à la révision des versions successives menant à la production de la version finale du manuscrit. Dominique Boucher, Élisabeth Déry et Jean-François Roux, co-chercheurs, ont été responsables du recrutement des superviseurs et des stagiaires dans leurs programmes respectifs en réadaptation. Ils ont participé à l'analyse de l'ensemble des données et à la révision des versions successives menant à la production de la version finale du manuscrit.

\section{Conflits d'intérêts}

Les auteurs déclarent n'avoir aucun lien d'intérêt concernant les données publiées dans cet article.

\section{Approbation éthique}

Le protocole de recherche a reçu l'approbation du comité d'éthique de la recherche de l'Université Laval (certificat no. 2016-121).

\section{Remerciements d'usage}

Les auteurs remercient le Fonds Gilles-Cormier de la Faculté de médecine de l'Université Laval pour son soutien financier, ainsi que les superviseurs et les stagiaires qui ont participé à l'étude.

\section{Annexe A: Guide d'entrevue sur l'alliance pédagogique en supervision clinique, utilisé pour les entrevues semi-structurées conduites au cours de l'étude}

L'interviewer demandait au participant de se remémorer des situations de supervision, en particulier des situations de rétroaction récentes ou plus anciennes qu'il avait vécues comme superviseur ou comme stagiaire (ou résident). Puis, il posait les questions suivantes, avec la possibilité d'ajouter des sous-questions de clarification au besoin :

Que signifie pour vous la notion d'alliance pédagogique lors de la rétroaction en supervision clinique?

Depuis que vous supervisez en...ou depuis que vous êtes stagiaire ou résident en... pouvez-vous me parler d'une ou de quelques situations de supervision où l'alliance pédagogique entre le superviseur et le stagiaire était satisfaisante?

Selon vous, qu'est-ce qui a le plus contribué à ce que ce soit satisfaisant?

Depuis que vous supervisez en... ou depuis que vous êtes stagiaire ou résident en..., avez-vous déjà vécu une ou des situations de supervision où l'alliance pédagogique entre le superviseur et le stagiaire était insatisfaisante?

Si oui : 
Pouvez-vous m'en parler.

Selon vous, qu'est-ce qui a contribué à ce que ce soit insatisfaisant?

Comment avez-vous géré cette (ces) situation (s)?

Avant de terminer cette entrevue, avez-vous d'autres commentaires à faire en lien avec l'alliance pédagogique lors de la rétroaction en supervision clinique?

\section{Références}

1. Kilminster SM, Cottrell D, Grant J, Jolly B. AMEE Guide no. 27: Effective educational and clinical supervision. Med Teach 2007;29:2-19.

2. Ramani S, Leinster S. AMEE Guide no. 34: Teaching in the clinical environment. Med Teach 2008;30:347-64.

3. Côté L, Perry G, Cloutier PA. Développer son modèle de rôle en formation pratique : la contribution d'une communauté de pratique de cliniciens enseignants. Pédagogie Médicale 2013;14:243-51.

4. Norcini J, Burch V. Workplace-based assessment as an educational tool: AMEE Guide no.31. Med Teach 2007;29:855-71.

5. Teunissen PW, Stapel DA, van der Vleuten CP, Scherpbier A, Boor K, Scheele F. Who wants feedback? An investigation of the variables influencing residents' feedback-seeking behavior in relation to night shifts. Acad Med 2009;84:910-17.

6. Mann KV, van der Vleuten CP, Eva KW, Armson H, Chesluk B, Dornan T, Holmboe E, Lockyer J, Loney E, Sargeant J. Tensions in informed self-assessment: how the desire for feedback and reticence to collect and use it can conflict. Acad Med 2011;86:1120-27.

7. Sargeant J, Eva KW, Armson H, Chesluk B, Dornan T, Holmboe E, Lockyer JM, Loney E, Mann KV, van der Vleuten CP. Features of assessment learners use to make informed self-assessments of clinical performance. Med Educ 2011;45:636-47.

8. Eva KW, Armson H, Holmboe E, Lockyer J, Loney E, Mann K, Sargeant J. Factors influencing responsiveness to feedback: on the interplay between fear, confidence, and reasoning processes. Adv Health Sci Educ Theory Pract 2012;17:15-26.

9. Watling, C, Driessen, E, van der Vleuten, CP, Lingard, L. Learning from clinical work: the roles of learning cues and credibility judgements. Med Educ 2012;46:192-200.

10. Watling C. Cognition, culture, and credibility: deconstructing feedback in medical education. Perspect Med Educ 2014;3:124-28.

11. van de Ridder JM, Berk FC, Stokking KM, ten Cate OTJ. 2015a. Feedback providers' credibility impacts students' satisfaction with feedback and delayed performance. Med Teach 37:767-74.

12. Telio S, Regehr G, Ajjawi R. Feedback and the educational alliance: examining credibility judgements and their consequences. Med Educ 2016;50:933-42.

13. Bowen, L, Marshall, M, Murdoch-Eaton, D. Medical student perceptions of feedback and feedback behaviors within the context of the "Educational alliance". Acad Med 2017;92:1303-12.
14. Ramani, S, Könings, KD, Mann, KV, van der Vleuten, CP. Uncovering the unknown: A grounded theory study exploring the impact of self-awareness on the culture of feedback in residency education. Med Teach 2017;39: 1065-73.

15. Archer JC. State of the science in health professional education: Effective feedback. Med Educ 2010;44:101-08.

16. Eva KW, Regehr G. Effective feedback for maintenance of competence: From data delivery to trusting dialogues. CMAJ 2013;185:463-64.

17. Sargeant J, Lockyer J, Mann KV, Holmboe E, Silver I, Armson $\mathrm{H}$ et al. Facilitated reflective performance feedback: developing an evidence and theory-based model that builds relationship, explores reactions and content, and coaches for performance change (R2C2). Acad Med 2015;90:1698-1706.

18. Telio S, Ajjawi R, Regehr G. The "educational alliance" as a framework for reconceptualizing feedback in medical education. Acad Med 2015;90:609-14.

19. Bordin ES. The generalizability of the psychoanalytic concept of the working alliance. Psychother Theory Res Pract 1979;16:252-60.

20. Bordin ES. A working alliance based model of supervision. Couns Psychol 1983;11:35-42.

21. Côté L. Réflexion sur une expérience de supervision clinique sous l'angle de l'alliance pédagogique. Pédagogie Médicale 2015; $16: 79-84$.

22. Hauer KE, ten Cate O, Boscardin C, Irby DM, Iobst W, O'Sullivan PS. Understanding trust as an essential element of trainee supervision and learning in the workplace. Adv Health Sci Educ Theory Pract 2014;19:435-56.

23. Hauer KE, Oza SK, Kogan JR, Stankiewicz CA, StenforsHayes T, ten Cate O, Batt J, O'Sullivan PS. How clinical supervisors develop trust in their trainees: a qualitative study. Med Educ 2015;49:783-95.

24. Strauss A, Corbin J. Basics of qualitative research: techniques and procedures for developing grounded theory. ThousandOaks, CA: Sage, 1998.

25. Watling CJ, Lingard L. Grounded theory in medical education research: AMEE Guide no. 70. Med Teach 2012;34:850-61.

26. Creswell JW. Qualitative inquiry and research design: Choosing among five approaches. Thousand Oaks (CA): Sage Publ., 2007.

27. Denzin NK. The research act: a theorical introduction to sociological methods. New York: McGraw-Hill, 1978.

28. Morse J. The significance of saturation. Qual Health Res 1995;5:147-49.

29. Paillé P. L'analyse par théorisation ancrée. Cahiers de recherche sociologique 1994;23:147-81.

30. Martin P, Coopley J, Tyack Z. Twelve tips for effective clinical supervision based on a narrative literature review and expert opinion, Med Teach 2014;36:201-207.

31. Bernard J, Goodyear RK. Fundamentals of clinical supervision. Upper Saddle River (NY): Pearson, 2014.

32. Bing-You R, Varaklis K, Hayes V, Trowbridge R, Kemp H, McKelvy D. The feedback tango: An integrative review and analysis of the content of the teacher-learner feedback exchange. Acad Med 2018;93:657-63.

33. Wearne S. Effective feedback and the educational alliance. Med Educ 2016;50:891-92.

Citation de l'article : Côté L., Breton E., Boucher D., Déry É., Roux J.-F., L'alliance pédagogique en supervision clinique : une étude qualitative en sciences de la santé. Pédagogie Médicale 2017:18;161-170 